\title{
Mechanical and Thermal Analysis of Classical Functionally Graded Coated Beam
}

\author{
Abdolreza Toudehdehghan ${ }^{1,2^{*}}, M d$. Mujibur Rahman ${ }^{2}$, and Faris Tarlochan $^{3}$ \\ ${ }^{1}$ Department of Mechanical Engineering, INTI International University, Jalan BBN 12/1, Bandar \\ Baru Nilai, 71800 Nilai, Negeri Sembilan, Malaysia \\ ${ }^{2}$ Department of Mechanical Engineering, Universiti Tenaga Nasional, km7, Jalan Kajang-Puchong, \\ 43009 Kajang, Selangor, Malaysia \\ ${ }^{3}$ Mechanical and Industrial Engineering, College of Engineering, Qatar University, P.O. Box 2713, \\ Doha, Qatar
}

\begin{abstract}
The governing equation of a classical rectangular coated beam made of two layers subjected to thermal and uniformly distributed mechanical loads are derived by using the principle of virtual displacements and based on Euler-Bernoulli deformation beam theory (EBT). The aim of this paper was to analyze the static behavior of clamped-clamped thin coated beam under thermo-mechanical load using MATLAB. Two models were considered for composite coated. The first model was consisting of ceramic layer as a coated and substrate which was metal (HC model). The second model was consisting of Functionally Graded Material (FGM) as a coated layer and metal substrate (FGC model). From the result it was apparent that the superiority of the FGC composite against conventional coated composite has been demonstrated. From the analysis, the stress level throughout the thickness at the interface of the coated beam for the FGC was reduced. Yet, the deflection in return was observed to increase. Therefore, this could cater to various new engineering applications where warrant the utilization of material that has properties that are well-beyond the capabilities of the conventional or yesteryears materials.
\end{abstract}

\section{Introduction}

Functionally Graded Materials (FGMs) were initially used as a thermal barrier by Japanese scientists in 1984. Since then, this kind of materials has been expanded and developed as a heat-resistant material. As an example, the surface temperature of the space shuttle is approximately $21000 \mathrm{~K}$, that the temperature difference between the outside to the inside reaches $16000 \mathrm{~K}$. Therefore, conventional composites cannot resist this temperature difference [1]. Unlike the conventional composites, in FGMs, a combination of materials is changed continuously from high resistance temperature materials such as ceramics on one side to the other side, which has strong mechanical toughness materials, such as metals. FGM is used not only as a thermal barrier but also as a corrosion and wear coating resistant

\footnotetext{
* Corresponding author: Abdolreza.toudehdehghan@newinti.edu.my
} 
[2]. Javaheri and Eslami [3] analyzed functionally graded rectangular plate under thermal loads based on higher order shear deformation theory and power law function for FGMs. Most of the researcher, in order to investigate on FGM structures, are used power law function [4-6]. Ho Chi and Chung [7] used power-law, exponential and sigmoid function on the classical plate theory and compared the results with applied the finite element method.

Generally, the surface of structures or elements is weaker than the inside of them. Therefore, the coating has been used for decades to increase the resistance of the surface of structures, reduce the stress concentration on the surface and stop cracks, which have been generated. In the conventional surface coating method, one layer as a coated will be covered the surface of the body and the thickness range is from a micrometer to several millimeters. The coating thickness usually is depended on the type of material which is used as a substrate layer [8]. For example, in Thermal Barrier Coating (TBC) one-layer ceramic is bonded to the substrate layer. The disadvantage of this type of coating is that due to the use of two completely different materials (ceramic-metal), the concentration of stress between the two layers' increases, and thus the two layers are separated [9]. To improve the weakness, FGMs have lately been suggested to modify the conventional coating [10]. Due to the characteristic of FGM composites, the properties of materials in the composite change very gradually between two different materials. This property makes the separation between the composite layers not present. Mainly, under a high-temperature atmosphere like a nozzle of the shuttle, nuclear fusion reactor, internal combustion engine and so on at the interface of two layers due to different thermal expansion, the mismatch will be happened [7]. Coating structures with FGMs are called FGC. FGC beams under mechanical, thermal and thermos-mechanical loads are studied by several of researches [11-17].

The present article, a static analysis of FGC beam is studied by using a series displacement as a linear combination of know function which satisfies the boundary conditions and unknown parameters. The governing equation of FGC beam based on classical beam displacement theory and principle of virtual is derived. The material properties of FG layer through the thickness is considered an exponential function. In order to demonstrate the behavioural difference of a conventional composite with a modern composite which is consisting of an FGM layer. Two composite models are considered. Lastly, the effect of the rectangular clamped-clamped beam under thermal and mechanical loads are investigated.

\section{Extracting governing equations}

The displacement field components in Euler-Bernoulli's beam are considered in the form below [18]:

$$
\begin{aligned}
& u(x, y, z)=u_{0}(x, y)-z \frac{d w_{0}(x)}{d x} \\
& V(x, y, z)=0 \\
& w(x, y, z)=w_{0}(x)
\end{aligned}
$$

Thus, the axial strain component in mid-plane direction of beam is 


$$
\varepsilon_{x x}=\frac{d u}{d x}=\frac{d u_{0}}{d x}-z \frac{d^{2} w_{0}}{d x^{2}}
$$

\subsection{FGMs material properties}

The continuous composition of FGMs can be described by using several mathematical models to show the material properties of the composite. The material properties of FGMs are inhomogeneous microscopically and change with changing temperature, therefore the material properties of FGMs depend on position and temperature. One of the mathematical models of material properties was used in a lot of research in order to study fracture mechanics, crack propagation, vibration, and bending, is exponential function $[7,11,19$ 26].

Material properties with exponential function of FGMs are:

$$
\begin{aligned}
& E_{f e}(z)=E_{c} e^{\left(\frac{1}{h} \ln \left(\frac{E_{m}}{E_{c}}\right)\left(z+\frac{h}{2}\right)\right)} \\
& \alpha_{f e}(z)=\alpha_{c} e^{\left(\frac{1}{h} \ln \left(\frac{\alpha_{m}}{\alpha_{c}}\right)\left(z+\frac{h}{2}\right)\right)} \\
& k_{f e}(z)=k_{c} e^{\left(\frac{1}{h} \ln \left(\frac{k_{m}}{k_{c}}\right)\left(z+\frac{h}{2}\right)\right)}
\end{aligned}
$$

\subsection{Governing equation of beam}

To derive the governing equations of the beam, we use the principle of virtual work defined as [27]:

$$
\delta W=\delta W_{I}+\delta W_{E}=0
$$

where $\delta W_{I}$ and $\delta W_{E}$ are virtual work cause of internal forces and virtual work cause of external forces respectively. The boundary conditions of clamped-clamped beam are

$$
w(0)=w(a)=\frac{d w}{d x}(0)=\frac{d w}{d x}(a)=0
$$

Also, the variation form of the beam should satisfy the equation (5):

$$
\delta w(0)=\delta w(a)=0 \quad \frac{d \delta w}{d x}(0)=\frac{d \delta w}{d x}(a)=0
$$

The stress-strain relation in the mid-plane direction under thermal and mechanical loading is

$$
\sigma_{x x}=E(z) \varepsilon_{x x}-E(z) \alpha(z) T(z)
$$

The total internal virtual work done is 


$$
\delta W_{I}=\int_{0}^{a} \int_{A} \sigma_{x x} \delta \varepsilon_{x x} d A d x
$$

The virtual work done by external distributed load by using the virtual displacement is

$$
\delta W_{E}=-\int_{0}^{a} b P_{0} . \delta w d x
$$

By using equations (8) and (9) into equation (4) and with consideration the boundary conditions (equations (5) and (6)), it can be obtained the governing equation of beam, taking into account Euler-Bernoulli theory, according to the following:

$$
\int_{0}^{a} D_{x x} \frac{d^{2} w}{d x^{2}} \frac{d^{2} \delta w}{d x^{2}} d x-\int_{0}^{a} P^{*}(x) \delta w d x-\int_{0}^{a} b P_{0} \delta w d x=0
$$

where $D_{x x}=I E_{f e}(z)$

\subsection{Equations of the temperature change cross the thickness of functionally graded beam}

Two models coated beam were considered. The first is conventional coated beam (HC model). In this model the coated beam has two different layers. The homogeneous ceramic layer $\left(h_{3}\right)$ as a thermal barrier coating and substrate as a homogeneous metal layer $\left(h_{1}\right)$ which are located from top to bottom of the model in thickness direction respectively. The second model is FGC beam (FGC model). FGC model has FGM layer $\left(\mathrm{h}_{2}\right)$ on top and substrate metal homogeneous $\left(\mathrm{h}_{1}\right)$.

By considering the temperature variation is happened just in the thickness direction of the structures and steady-state heat condition. The heat conduction equation through the thickness of the beam is as following [28]:

$$
-\frac{d}{d z}\left[k(z) \frac{d T}{d z}\right]=0
$$

\subsubsection{Temperature equations of homogeneous layers of beam}

To find the temperature function across the thickness of homogeneous single layer has to use thermal conductivity coefficient of homogeneous material and boundary condition across the thickness of the layer into the equation (11). Thermal equation across the thickness of the homogeneous ceramic coated layer and homogeneous metal substrate respectively will be as below:

$$
\begin{aligned}
& T_{c}(z)=\frac{1}{h_{2}}\left(\left(T_{m}-T_{u}\right) z+\left(\frac{h_{1}+h_{2}}{2}\right) T_{m}+\left(\frac{h_{2}-h_{1}}{2}\right) T_{u}\right) \\
& T_{s}(z)=\frac{1}{h_{1}}\left(\left(T_{L}-T_{m}\right) z-\frac{h_{2}-h_{1}}{2} T_{L}+\frac{h_{2}+h_{1}}{2} T_{m}\right)
\end{aligned}
$$


Nonlinear thermal equation across the thickness of FGM layer based on exponential function is as follows:

$$
\begin{gathered}
T_{f e}(z)=T_{u}+\left(T_{m}-T_{u}\right) \eta_{E}(z) \\
\eta_{E}(z)=\frac{1}{C_{E}}\left[\left(\frac{z+\frac{h}{2}}{h_{2}}\right)-\frac{1}{2 !} \ln \left(\frac{k_{m}}{k_{c}}\right)\left(\frac{z+\frac{h}{2}}{h_{2}}\right)^{2}+\frac{1}{3 !}\left(\ln \left(\frac{k_{m}}{k_{c}}\right)\right)^{2}\left(\frac{z+\frac{h}{2}}{h_{2}}\right)^{3}\right. \\
\left.-\frac{1}{4 !}\left(\ln \left(\frac{k_{m}}{k_{c}}\right)\right)^{3}\left(\frac{z+\frac{h}{2}}{h_{2}}\right)^{4}+\frac{1}{5 !}\left(\ln \left(\frac{k_{m}}{k_{c}}\right)\right)^{4}\left(\frac{z+\frac{h}{2}}{h_{2}}\right)^{5}-\frac{1}{6 !}\left(\ln \left(\frac{k_{m}}{k_{c}}\right)\right)^{5}\left(\frac{z+\frac{h}{2}}{h_{2}}\right)^{6}\right] \\
C_{E}=1-\frac{1}{2 !} \ln \left(\frac{k_{m}}{k_{c}}\right)+\frac{1}{3 !}\left(\ln \left(\frac{k_{m}}{k_{c}}\right)\right)^{2}-\frac{1}{4 !}\left(\ln \left(\frac{k_{m}}{k_{c}}\right)\right)^{3}+\frac{1}{5 !}\left(\ln \left(\frac{k_{m}}{k_{c}}\right)\right)^{4}-\frac{1}{6 !}\left(\ln \left(\frac{k_{m}}{k_{c}}\right)\right)^{5}
\end{gathered}
$$

\subsection{Solution to rectangular coated beam}

An approximate solution, the deflection of the beam will be obtained based on boundary conditions (equation (6)) and geometry which is occupying the space defined by

$$
0 \leq x \leq a \quad-\frac{h}{2} \leq z \leq \frac{h}{2}
$$

Where $a$ and $h$ are the length of the beam and the total thickness of beam included the two layers respectively.

To find the answer to equation (10), based on boundary condition and geometry of beam, an approximate solution is obtained as:

$$
w=\sum_{i=1}^{m} A_{i} \sin ^{2}\left(\frac{i \pi x}{a}\right)
$$

The thermal load $P^{*}(x)$ in equation (10) by using Fourier series and some simplification will be as:

$$
\begin{gathered}
P_{x}^{*}(x)=\sum_{m=1}^{\infty} \frac{4 m \pi}{a^{2}} M_{T} \sin \left(\frac{m \pi x}{a}\right) \\
m=1,3,5, \ldots
\end{gathered}
$$

By using the solution equation, variation form of solution and thermal load in equation (10) we get

$$
R_{(\gamma)(i)} A_{i}-F_{\gamma}=0
$$

\subsection{Numerical calculations}

As mentioned in section 2.3, HC and FGC models have two layers with the same thickness. Therefore, the thickness of the coated layer and metal substrate are $4 \mathrm{~mm}$ and $6 \mathrm{~mm}$ respectively. In order to find the material properties which are defined as the exponential functions in equation (3). The modulus of elasticity, the thermal expansion coefficient, and thermal conductivity were assumed as follow: 
$\mathrm{E}_{\mathrm{c}}=100 \mathrm{GPa}, \mathrm{E}_{\mathrm{m}}=10 \mathrm{GPa}, \alpha_{c}=1.4 \times 10^{-5}\left({ }^{o} K^{-1}\right), \alpha_{m}=0.9 \times 10^{-5}\left({ }^{o} K^{-1}\right)$

$\mathrm{k}_{\mathrm{c}}=40 \mathrm{~W} /\left(\mathrm{m} .{ }^{\circ} \mathrm{K}\right), \mathrm{k}_{\mathrm{m}}=90 \mathrm{~W} /\left(\mathrm{m} .{ }^{\circ} \mathrm{K}\right)$

The Poisson's ratio was assumed constant and equal to $v=0.3$.

\subsubsection{Static analysis}

The dimensions of the coated beam in both models were taken as $\mathrm{a}=50 \mathrm{~cm}, \mathrm{~b}=5 \mathrm{~cm}$, and $\mathrm{h}$ $=1 \mathrm{~cm}$. On top of the coated beam with clamped-clamped boundary conditions (equation (5)), the uniformly distributed load and temperature are assumed to be $P_{0}=22000 \mathrm{kPa}$ and $T_{u}=1000^{\circ} \mathrm{K}$ respectively. The coordinate axes were located in the middle of the volume of the rectangular coated beam. Only bending behaviour of the coated beam was considered, thus displacement of mid-plane along x-axis equal to zero. The temperature of the bottom surface of the coated beam was assumed $T_{L}=300^{\circ} \mathrm{K}$. Also in equation (18), $\gamma$ and $i$ were considered equal to two.

Fig. 1 shows deflection of the centre of the coated beam for both models. The deflection of FGC model much bigger than HC model, because of the ceramic percentage in FGC model is less than HC model. Fig. 2 shows the stress variation throughout the thickness of the coated beam for both models. The graph of the HC model clearly demonstrates the stress singularity at the interface of the metal substrate and homogeneous ceramic coated layer. Therefore, this phenomenon causes to create a de-bonding at the interface of two layers. Vice versa for FGC mode, the graph shows the stress gradually changed at the interface of two layers. Thus stress singularity and concentration will decrease.

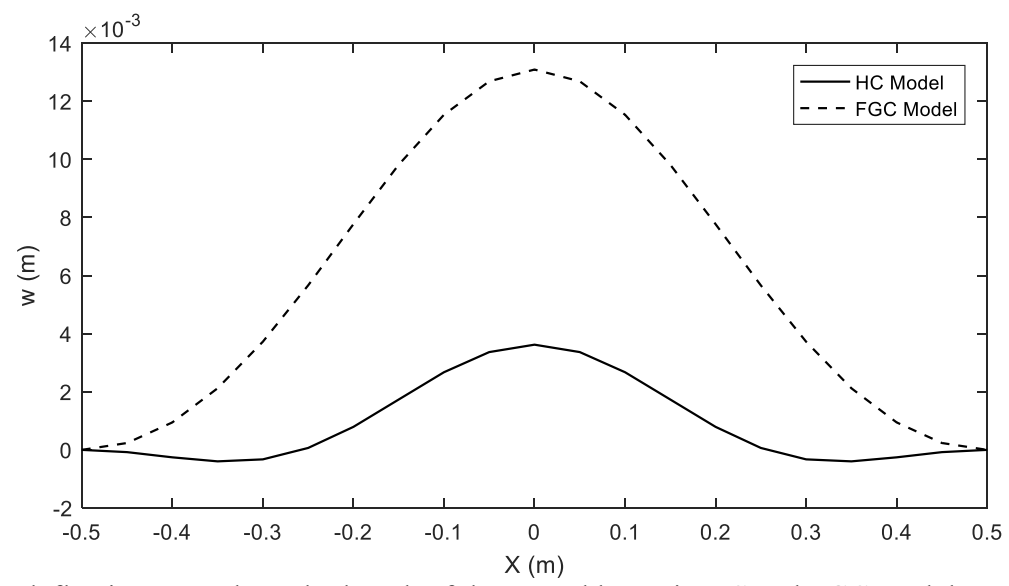

Fig. 1. The deflection $(w)$ along the length of the coated beam in HC and FGC model. 


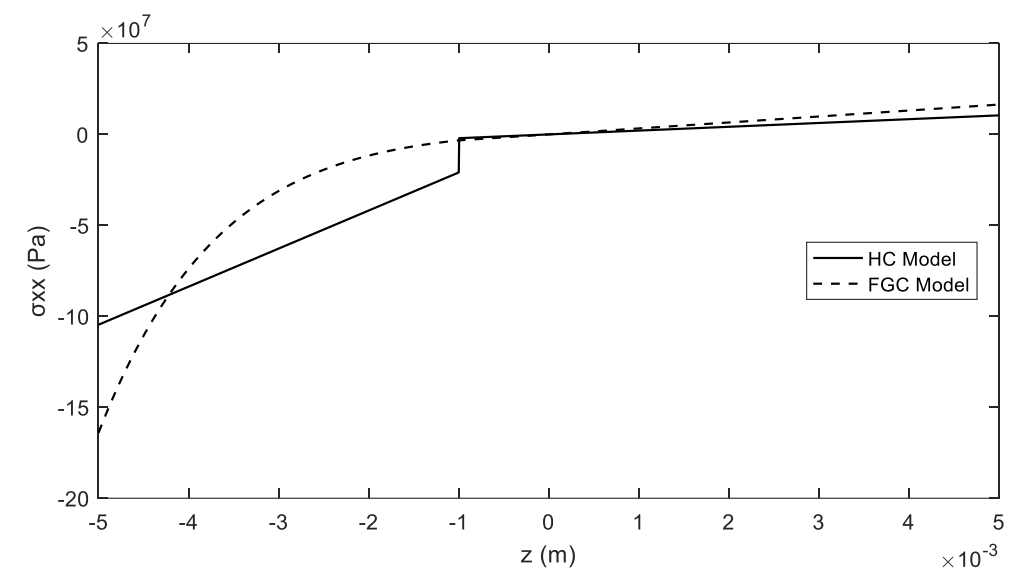

Fig. 2. The stress $\left(\sigma_{x x}\right)$ throughout the thickness of centre of the coated beam in HC and FGC model.

\section{Conclusions}

The static analysis of conventional coated and FGC rectangular beam under the transverse distributed load and thermal excitation due to the thermal environment. The assumption of position dependent material properties was considered as an exponential function. The governing equation is derived based on the Euler-Bernoulli theory and exponential function for FGM layer by using the virtual work principle. Transverse displacement and stress throughout the thickness of the beam are considered. With obtained an approximation function which is supported clamped-clamped boundary conditions, the displacement or behaviour of the beam structure and stress throughout the thickness under thermal and mechanical loads are found. As noted initially, the HC model will experience delamination upon the introduction of stress to the model. Hence, this study has successfully demonstrated the superiority of FGC composite against conventional coated composite. From the analysis, the stress level throughout the thickness at the interface of the coated beam for the FGC was reduced. Yet, the deflection in return was observed to increase. Therefore, this could cater to various new engineering applications where warrant the utilization of material that has properties that are well-beyond the capabilities of the conventional or yesteryears materials.

\section{References}

1. M. Koizumi, Composites Part B: Engineering 28 1-4, (1997)

2. S. Suresh, A. Mortensen, Fundamentals of functionally graded materials (The Institut of Materials, 1998)

3. R. Javaheri, M. Eslami, Journal of thermal stresses 25, 603-625 (2002)

4. W. Zhang, Y. Hao, X. Guo, L. Chen, Meccanica 47, 985-1014 (2012)

5. Y. Hao, W. Zhang, J. Yang, S. Li, Journal of Mechanical Science and Technology 25, 1637-1646 (2011)

6. R. Javaheri, M. Eslami, AIAA Journal 40, 162-184 (2002)

7. S.H. Chi, Y.L. Chung, International Journal of Solids and Structures 43, 3657-3674 (2006)

8. J. Mencik, Mechanics of components with treated or coated surfaces 42 (Springer Science \& Business Media, 2013) 
9. Y.L. Chung, C.F. Pon, International Journal of Solids and Structures 38, 75-90 (2001)

10. M. Kashtalyan, M. Menshykova, International Journal of Solids and Structures 44, 5272-5288 (2007)

11. B. Sankar, Composites Science and Technology 61, 689-696 (2001)

12. R. Shahsiah, K. Nikbin, M. Eslami, Iranian Journal of Mechanical Engineering 10, 64-80 (2009)

13. A. Davoodinik, G. Rahimi, Acta Mechanica Sinica 27, 767-777 (2011)

14. G. Rahimi, A. Davoodinik, IUST International Journal of Engineering Science 19, 105-113 (2008)

15. S. Esfahani, Y. Kiani, M. Eslami, International Journal of Mechanical Sciences 69, 10-20 (2013)

16. B. V. Sankar, J. T. Tzeng, AIAA journal 40, 1228-1243 (2002)

17. L. Ma and D. Lee, Composite Structures 93, 831-842 (2011)

18. C. Wang, J. N. Reddy, K. Lee, Elsevier (2000)

19. F. Erdogan, Composites Engineering 5, 753-770 (1995)

20. J. Yang, Y. Chen, Composite Structures 83, 48-60 (2008)

21. F. Erdogan, A. C. Kaya, P. F. Joseph, Journal of Applied Mechanics, 58, 410-418, (1991)

22. W. B H, Journal of applied Mechanics 64, 449 (1997)

23. H. J. Choi, Journal of Applied Mechanics 63, 479-486 (1996)

24. Z.H. Jin, N. Noda, Journal of Applied Mechanics 61, 738-740 (1994)

25. N. Noda, Z.H. Jin, JSME international journal. Ser. A, Mechanics and material engineering 38, 364-369 (1995)

26. B. Wang, J. Han, S. Du, Journal of applied mechanics 67, 87-95 (2000)

27. J. N. Reddy, Energy principles and variational methods in applied mechanics (John Wiley \& Sons, 2017)

28. H.S. Shen, Functionally graded materials: nonlinear analysis of plates and shells (CRC press, 2016) 